\title{
Transportation and Material Moving Occupations
}

National Cancer Institute

\section{Source}

National Cancer Institute. Transportation and Material Moving Occupations. NCI

Thesaurus. Code C97645.

A class of professional or vocational positions of employment that involve transportation or material moving. 